\title{
Transnitrosation of Nitrosothiols: characterization of an elusive intermediate
}

\author{
Supporting information
}

Laura L. Perissinotti ${ }^{\star}$, Adrián G. Turjanski, Darío A. Estrin and Fabio Doctorovich

Departamento de Química Inorgánica, Analítica y Química Física / INQUIMAE, Facultad de Ciencias Exactas y Naturales, Universidad de Buenos Aires. Ciudad Universitaria, Pabellón II, (C1428EHA) Buenos Aires, Argentina. 


\section{Materials and methods}

Materials. L-cysteine ethyl ester (ECyS) was purchased from Sigma Aldrich and used as received, S-nitroso-L-cysteine ethyl ester (ECySNO) was prepared as reported in the literature. ${ }^{1}$ ECyS ${ }^{15} \mathrm{NO}$ was prepared in an analogous manner to ECySNO, using $\mathrm{Na}^{15} \mathrm{NO}_{2}$ purchased form Aldrich.

Methanol was purchased from Cicarelli, it was dried and distilled. $\mathrm{CD}_{3} \mathrm{OD}$ and $\mathrm{D}_{2} \mathrm{O}$ were purchased from Aldrich and used as received. The water used in all reactions was MilliQ Water obtained from deionized water.

Unless otherwise noted, all manipulations were performed with exclusion of oxygen using standard Schlenk procedures or septa; the inert gas used was Argon or $\mathrm{N}_{2}$.

Kinetics. All rate measurements were carried out in water at different temperatures in a range of 30 degrees, in $0.5 \mathrm{M}$ phosphate buffer ( $\mathrm{pH} 7.4)$ and EDTA $(10 \mathrm{mM})$. $\mathrm{pH}$ was measured with a Metrohm $744 \mathrm{pH}$ Meter. $\mathrm{pH}$ of ECyS and ECySNO solutions was adjusted to 7.4 with diluted KOH. A fresh solution of ECySNO was prepared immediately before each experiment, and the concentration was confirmed spectrophotometrically by measuring the absorbance at $344 \mathrm{~nm}$. The ECySNO concentration was fixed at $1 \times 10^{-3} \mathrm{M}$ and ECyS at $0.1 \mathrm{M}$. Reactions were followed spectrophotometrically at $344 \mathrm{~nm}$, by measuring the disappearance of the absorbance due to ECySNO. In all the experiments the thiol was in at least a 100-fold excess over the nitrosothiol. Excellent first order behavior occurred throughout.

The data were collected directly from the spectrophotometer to a PC and commercial software was used to obtain values of the first order rate constants. Values of $\mathrm{k}$ were reproducible at every temperature.

NMR Spectroscopy. NMR spectra were acquired on a Bruker AM 500 spectrometer. ${ }^{1} \mathrm{H}$ NMR and ${ }^{15} \mathrm{~N}$ NMR spectra were acquired for the reaction in situ. ${ }^{15} \mathrm{~N}$ NMR spectra were recorded at 50.68 $\mathrm{MHz}, 6150$ transients were collected with 5-s relaxation delay and referenced to an external standard of $\mathrm{Na}^{15} \mathrm{NO}_{2}$ at $232 \mathrm{ppm}$. All measurements were carried out in methanol. Both, ECySNO and ECyS concentration were fixed at $0.1 \mathrm{M}$. All the solutions were manipulated in a MBraun drybox with a nitrogen atmosphere containing less than $10 \mathrm{ppm}$ of water and oxygen.

UV/Visible Spectroscopy. Absorption spectra were acquired on a Hewlett-Packard HP8453 diode array spectrometer equipped with a RC6 LAUDA thermostat.

Theoretical Calculations. All the calculations performed in this work were carried out using the Gaussian $98^{2}$ package. We have fully optimized the geometries of all species at the B3LYP ${ }^{3}$ level with a $6-311+\mathrm{G}^{*}$ basis set without symmetry constraints. Each stationary point in the gas phase was characterized by performing a normal modes analysis. The transition state was determined by using the synchronous transit-guided quasi-Newton algorithm developed by Schlegel et al. ${ }^{4}$

Free energy differences in the gas phase were obtained from the isolated species QM geometry optimization and normal modes calculations by using standard statistical mechanics techniques, in the approximation of ideal gas, rigid rotor and harmonic vibrational motions. As usual the imaginary frequency was not taken into account in the transition state calculations. The relevant species exhibited several very low frequency vibrational modes, for which the harmonic approximation may lead to significant errors. ${ }^{5}$ Dealing with these low frequency motions in an appropriate way is far from trivial, and is probably not worth the effort, considering that additional errors may arise from the fact that the reaction proceeds in solution, and not in gas phase.

Solvent effects were modeled using the polarized continuum model (PCM) scheme. The $\mathrm{PCM}^{6}$ implementation, in which the self-consistency between the solute wave function and solvent polarization is achieved during the self consistent field cycle, has been employed.

Despite the previously mentioned flaws, the analysis of the computed free energy results yields some qualitative insight about the reaction mechanism. We obtained $\Delta G^{0}$ and $\Delta G^{\#}$ values of 14.3 and $30.7 \mathrm{kcal} / \mathrm{mol}$, including also solvation effects. The latter value compares reasonably well with the experimental value of $\Delta \mathrm{G}^{\#}$ of $25 \mathrm{kcal} / \mathrm{mol}$ in aqueous solution estimated using transition state theory. 


\section{Cartesian coordinates from B3LYP/6-311+G* optimizations:}

\begin{tabular}{|c|c|c|c|c|}
\hline \multirow{2}{*}{$\begin{array}{l}\text { Center } \\
\text { Number }\end{array}$} & \multirow{2}{*}{$\begin{array}{l}\text { Atomic } \\
\text { Number }\end{array}$} & \multicolumn{3}{|c|}{ Coordinates (Angstroms) } \\
\hline & & $\mathrm{X}$ & Y & $\mathrm{Z}$ \\
\hline 1 & 8 & -1.135600 & 1.107775 & 1.001861 \\
\hline 2 & 8 & -1.443289 & -0.698550 & -0.317524 \\
\hline 3 & 6 & -0.702028 & 0.344239 & 0.165247 \\
\hline 4 & 6 & 0.692629 & 0.338948 & -0.403581 \\
\hline 5 & 1 & 0.660604 & -0.099457 & -1.404126 \\
\hline 6 & 6 & 1.593957 & -0.598540 & 0.472706 \\
\hline 7 & 1 & 1.524740 & -0.253068 & 1.513526 \\
\hline 8 & 1 & 1.168827 & -1.608262 & 0.431595 \\
\hline 9 & 16 & 3.340982 & -0.607307 & -0.087362 \\
\hline 10 & 7 & 1.182687 & 1.713966 & -0.513575 \\
\hline 11 & 1 & 1.062748 & 2.166134 & 0.391295 \\
\hline 12 & 1 & 2.193853 & 1.611992 & -0.644511 \\
\hline 13 & 6 & -2.753365 & -0.884314 & 0.240736 \\
\hline 14 & 1 & -2.946750 & -1.954880 & 0.141811 \\
\hline 15 & 1 & -2.733569 & -0.622650 & 1.300298 \\
\hline 16 & 6 & -3.801752 & -0.067634 & -0.500958 \\
\hline 17 & 1 & -4.798961 & -0.277189 & -0.098043 \\
\hline 18 & 1 & -3.606350 & 1.000603 & -0.392351 \\
\hline 19 & 1 & -3.805190 & -0.314060 & -1.566264 \\
\hline
\end{tabular}

2

\begin{tabular}{|c|c|c|c|c|}
\hline \multirow{2}{*}{$\begin{array}{l}\text { Center } \\
\text { Number }\end{array}$} & \multirow{2}{*}{$\begin{array}{l}\text { Atomic } \\
\text { Number }\end{array}$} & \multicolumn{3}{|c|}{ Coordinates (Angstroms) } \\
\hline & & $\mathrm{X}$ & $\mathrm{Y}$ & $\mathrm{Z}$ \\
\hline 1 & 8 & -1.490268 & -2.337501 & 0.478430 \\
\hline 2 & 7 & -2.048697 & -1.898204 & -0.457502 \\
\hline 3 & 16 & -2.253271 & -0.034593 & -0.543518 \\
\hline 4 & 6 & -1.384101 & 0.512773 & 0.962306 \\
\hline 5 & 1 & -2.100173 & 1.044257 & 1.588838 \\
\hline 6 & 1 & -1.070485 & -0.402327 & 1.475154 \\
\hline 7 & 6 & -0.179442 & 1.431957 & 0.699402 \\
\hline 8 & 7 & -0.609704 & 2.711890 & 0.161672 \\
\hline 9 & 1 & 0.267612 & 1.620198 & 1.683811 \\
\hline 10 & 6 & 0.914384 & 0.739408 & -0.135432 \\
\hline 11 & 8 & 1.392524 & 1.198200 & -1.143046 \\
\hline 12 & 8 & 1.283360 & -0.427128 & 0.423030 \\
\hline 13 & 1 & 0.169829 & 3.360999 & 0.134518 \\
\hline 14 & 1 & -0.915019 & 2.605907 & -0.802174 \\
\hline 15 & 6 & 2.326143 & -1.194246 & -0.239597 \\
\hline 16 & 1 & 2.119702 & -2.224027 & 0.051596 \\
\hline 17 & 1 & 2.198646 & -1.092484 & -1.317467 \\
\hline 18 & 6 & 3.708578 & -0.752433 & 0.204924 \\
\hline 19 & 1 & 4.464882 & -1.397227 & -0.252337 \\
\hline 20 & 1 & 3.913250 & 0.274805 & -0.101804 \\
\hline 21 & 1 & 3.814615 & -0.825739 & 1.290028 \\
\hline
\end{tabular}




\begin{tabular}{|c|c|c|c|c|}
\hline \multirow{2}{*}{$\begin{array}{l}\text { Center } \\
\text { Number }\end{array}$} & \multirow{2}{*}{$\begin{array}{l}\text { Atomic } \\
\text { Number }\end{array}$} & \multicolumn{3}{|c|}{ Coordinates (Angstroms) } \\
\hline & & $\mathrm{X}$ & Y & $\mathrm{Z}$ \\
\hline 1 & 6 & 2.235206 & -0.848989 & -0.940006 \\
\hline 2 & 16 & 1.444944 & -2.471240 & -1.097277 \\
\hline 3 & 7 & 0.134420 & -2.175497 & 0.882334 \\
\hline 4 & 8 & 0.177639 & -1.035973 & 1.185692 \\
\hline 5 & 16 & -1.911788 & -2.731958 & 0.158242 \\
\hline 6 & 6 & -2.421450 & -1.024844 & -0.134636 \\
\hline 7 & 1 & 2.223003 & -0.331200 & -1.904450 \\
\hline 8 & 1 & 1.623269 & -0.254488 & -0.241899 \\
\hline 9 & 1 & -1.601950 & -0.394508 & 0.251153 \\
\hline 10 & 1 & -2.501925 & -0.821204 & -1.207030 \\
\hline 11 & 6 & -3.726110 & -0.597805 & 0.562039 \\
\hline 12 & 6 & 3.699774 & -0.910841 & -0.403245 \\
\hline 13 & 1 & 4.305917 & -1.430030 & -1.148546 \\
\hline 14 & 7 & 3.859867 & -1.607574 & 0.875037 \\
\hline 15 & 6 & 4.250534 & 0.499061 & -0.333654 \\
\hline 16 & 8 & 3.918024 & 1.115127 & 0.829470 \\
\hline 17 & 8 & 4.862928 & 1.055222 & -1.218505 \\
\hline 18 & 7 & -4.886384 & -1.275359 & -0.024497 \\
\hline 19 & 1 & -3.666395 & -0.875299 & 1.615975 \\
\hline 20 & 6 & -3.850471 & 0.926590 & 0.536970 \\
\hline 21 & 8 & -3.999762 & 1.398118 & -0.730483 \\
\hline 22 & 8 & -3.832343 & 1.650864 & 1.507016 \\
\hline 23 & 1 & -5.752337 & -0.945324 & 0.390634 \\
\hline 24 & 1 & -4.931309 & -1.096879 & -1.022846 \\
\hline 25 & 1 & 3.332055 & -1.111066 & 1.588735 \\
\hline 26 & 1 & 3.391685 & -2.507285 & 0.763895 \\
\hline 27 & 6 & -4.082304 & 2.827271 & -0.903912 \\
\hline 28 & 6 & 4.311925 & 2.490506 & 0.993622 \\
\hline 29 & 1 & -4.645185 & 2.956488 & -1.830444 \\
\hline 30 & 1 & -4.652697 & 3.253586 & -0.076768 \\
\hline 31 & 6 & -2.702734 & 3.458155 & -1.003708 \\
\hline 32 & 1 & -2.795849 & 4.529821 & -1.209099 \\
\hline 33 & 1 & -2.150232 & 3.337506 & -0.070506 \\
\hline 34 & 1 & -2.123091 & 3.004198 & -1.810841 \\
\hline 35 & 1 & 4.413845 & 2.617317 & 2.073068 \\
\hline 36 & 1 & 5.284935 & 2.640313 & 0.522830 \\
\hline 37 & 6 & 3.271242 & 3.441131 & 0.423297 \\
\hline 38 & 1 & 3.558956 & 4.477923 & 0.628330 \\
\hline 39 & 1 & 3.183838 & 3.319643 & -0.657873 \\
\hline 40 & 1 & 2.292091 & 3.262379 & 0.874006 \\
\hline
\end{tabular}

4

\begin{tabular}{|c|c|c|c|c|}
\hline \multirow{2}{*}{$\begin{array}{l}\text { Center } \\
\text { Number }\end{array}$} & \multirow{2}{*}{$\begin{array}{l}\text { Atomic } \\
\text { Number }\end{array}$} & \multicolumn{3}{|c|}{ Coordinates (Angstroms) } \\
\hline & & $\mathrm{X}$ & $\mathrm{Y}$ & $\mathrm{Z}$ \\
\hline 1 & 6 & -1.583407 & 3.308859 & 0.362281 \\
\hline 2 & 6 & -3.051520 & 3.238332 & -0.033405 \\
\hline 3 & 8 & -3.423580 & 1.863903 & -0.415787 \\
\hline 4 & 6 & -3.781569 & 0.986390 & 0.597044 \\
\hline 5 & 8 & -3.862386 & 1.356535 & 1.777087 \\
\hline 6 & 6 & -4.060014 & -0.420604 & 0.083544 \\
\hline
\end{tabular}




$\begin{array}{ccrrr}7 & 7 & -5.150941 & -0.451902 & -0.896979 \\ 8 & 6 & -2.763901 & -1.032855 & -0.490308 \\ 9 & 16 & -2.828915 & -2.884687 & -0.778352 \\ 10 & 8 & 0.244145 & -1.244835 & 1.018635 \\ 11 & 7 & 0.381168 & -2.453890 & 1.006499 \\ 12 & 16 & 1.970645 & -3.072709 & -0.147721 \\ 13 & 6 & 2.556831 & -1.355336 & -0.602931 \\ 14 & 6 & 3.795552 & -0.890852 & 0.217002 \\ 15 & 6 & 4.163610 & 0.511619 & -0.219399 \\ 16 & 8 & 5.028128 & 0.790994 & -1.057809 \\ 17 & 7 & 3.644868 & -0.938950 & 1.674916 \\ 18 & 8 & 3.373458 & 1.462847 & 0.377763 \\ 19 & 6 & 3.583091 & 2.879693 & 0.010370 \\ 20 & 6 & 2.788562 & 3.241667 & -1.234866 \\ 21 & 1 & 2.780412 & -1.359329 & -1.668699 \\ 22 & 1 & 1.706557 & -0.692595 & -0.402545 \\ 23 & 1 & -1.950480 & -0.847170 & 0.220421 \\ 24 & 1 & -2.508469 & -0.510513 & -1.414500 \\ 25 & 1 & 4.646131 & -1.511478 & -0.061936 \\ 26 & 1 & -4.357248 & -0.996441 & 0.959197 \\ 27 & 1 & -6.025358 & -0.056842 & -0.577735 \\ 28 & 1 & -4.886822 & -0.127012 & -1.817304 \\ 29 & 1 & 2.950120 & -0.281918 & 2.010723 \\ 30 & 1 & 3.432331 & -1.879644 & 1.988033 \\ 31 & 1 & -3.260996 & 3.823022 & -0.927112 \\ 32 & 1 & -3.699062 & 3.560291 & 0.781812 \\ 33 & 1 & -1.299314 & 4.344347 & 0.572607 \\ 34 & 1 & -1.395710 & 2.714061 & 1.255478 \\ 35 & 1 & -0.949879 & 2.931904 & -0.441440 \\ 36 & 1 & 3.238505 & 3.422465 & 0.887274 \\ 37 & 1 & 4.651033 & 3.040909 & -0.131762 \\ 38 & 1 & 2.891124 & 4.310352 & -1.443567 \\ 39 & 1 & 3.152036 & 2.690351 & -2.101582 \\ 40 & 1 & 1.730200 & 3.019729 & -1.096368 \\ -------------------------------------------------------- \\ & & & & \\ 17 & & & \\ 1 & 1 & & & \end{array}$

Table SI 1. . Selected geometrical parameters for all species in vacuo calculated by density functional theory at the B3LYP/ 6-311+G* level.

\begin{tabular}{ccccccc}
\hline & MeSNO & ECySNO & $(\mathrm{MeS})_{2} \mathrm{NO}$ & $(\mathrm{ECyS})_{2} \mathrm{NO}$ & $\begin{array}{c}\text { TS } \\
\text { MeS } / \text { MeSNO }\end{array}$ & $\begin{array}{c}\text { TS } \\
\text { ECyS }^{-} / \text {ECySNO }\end{array}$ \\
\hline S2-N & 1.862 & 1.877 & 2.321 & 2.397 & 1.946 & 2.059 \\
S1-N & & & 2.325 & 2.241 & 3.761 & 3.691 \\
S-S & & & 3.696 & 3.642 & 4.122 & 4.844 \\
O-N-S2 & \multirow{2}{*}{117.3} & \multirow{2}{*}{116.6} & 108.8 & 110.8 & 114.8 & 113.019 \\
O-N-S1 & & & 108.7 & 108.1 & 81.8 & 91.308 \\
N-S2-C2 & \multirow{2}{*}{101.9} & \multirow{2}{*}{101.6} & 93.8 & 94.3 & 98.1 & 95.871 \\
N-S1-C1 & & & 93.9 & 93.2 & 75.5 & 77.334 \\
\hline
\end{tabular}




\section{Figure SI 1. ${ }^{1}$ H NMR spectra}

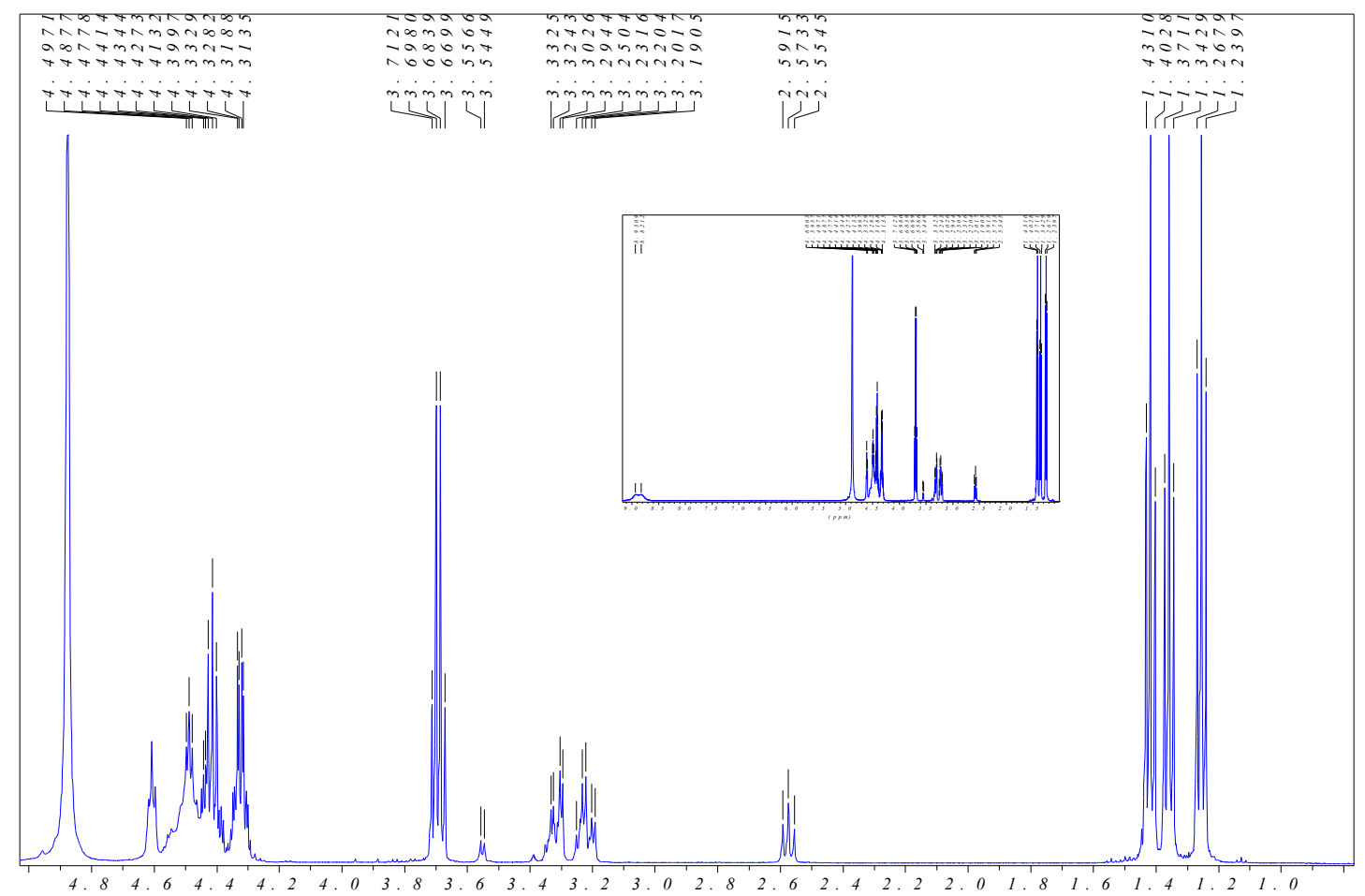

\section{${ }^{1}$ H NMR assignment:}

\section{1}

$\delta 1.43$ (triplet, $3 \mathrm{H}, \mathrm{CH}_{3}$ ); $\delta 3.29$ (doublet, $2 \mathrm{H}, \mathrm{CH}_{2}$ ) ECySH; $\delta 4.31-4.32$ (cuartet, $2 \mathrm{H}$, $\mathrm{OCH}_{2}$ ); $\delta 4.5$ (triplet, $1 \mathrm{H}, \mathrm{NCH}$ ); $\delta 2.57$ (triplet, $\left.1 \mathrm{H}, \mathrm{SH}\right) ; \delta 8.82(\mathrm{NH})$.

2

$\delta 1.37$ ( triplet , $\mathrm{H}, \mathrm{CH}_{3}$ ); $\delta$ 4.4-4.47 (multiplet, $3 \mathrm{H}, \mathrm{NCH}$ and $\mathrm{OCH}_{2}$ ); $\delta 4.6$ (triplet, $1 \mathrm{H}$, $\mathrm{NCH}) ; \delta 8.93(\mathrm{NH})$.

$\delta 1.25$ ( triplet , $\mathrm{H}, \mathrm{CH}_{3}$ ) ethanol; $\delta 3.68$ (cuartet, $4 \mathrm{H}, \mathrm{CH}_{2}$ ) ethanol 
Table SI 2. S-CH $\mathrm{CH}_{2}$ Signal deconvolution

\begin{tabular}{cc}
\hline ECyS/ECySNO & \% Intermediate \\
\hline 0.54 & 6.29 \\
1.08 & 19.59 \\
2.29 & 67.07 \\
2.78 & 92.65 \\
\hline
\end{tabular}

\section{Figure SI $2 .{ }^{13} \mathrm{C}$ NMR spectra}

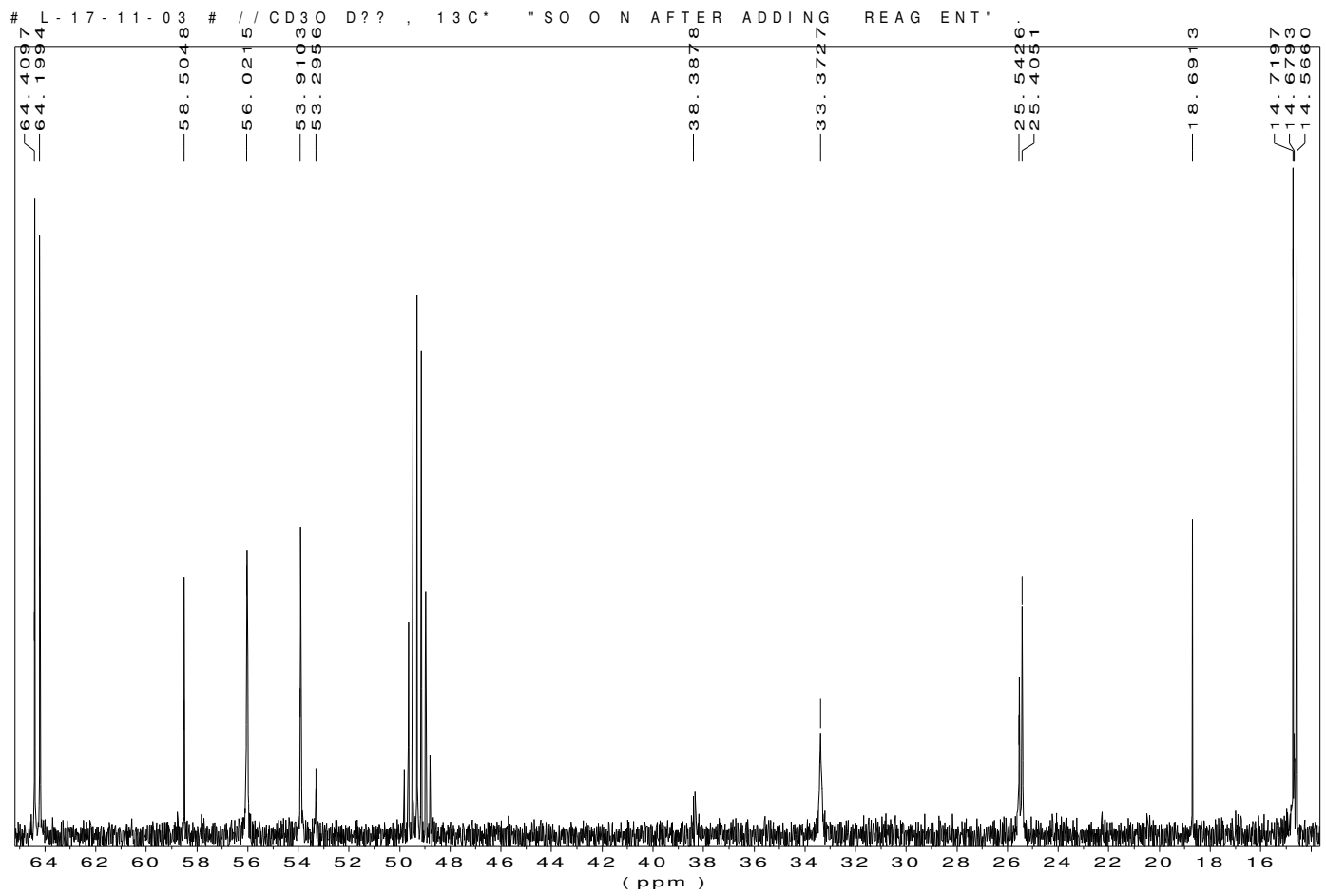


Figure SI 3. ${ }^{13} \mathrm{C}$ NMR DEPT

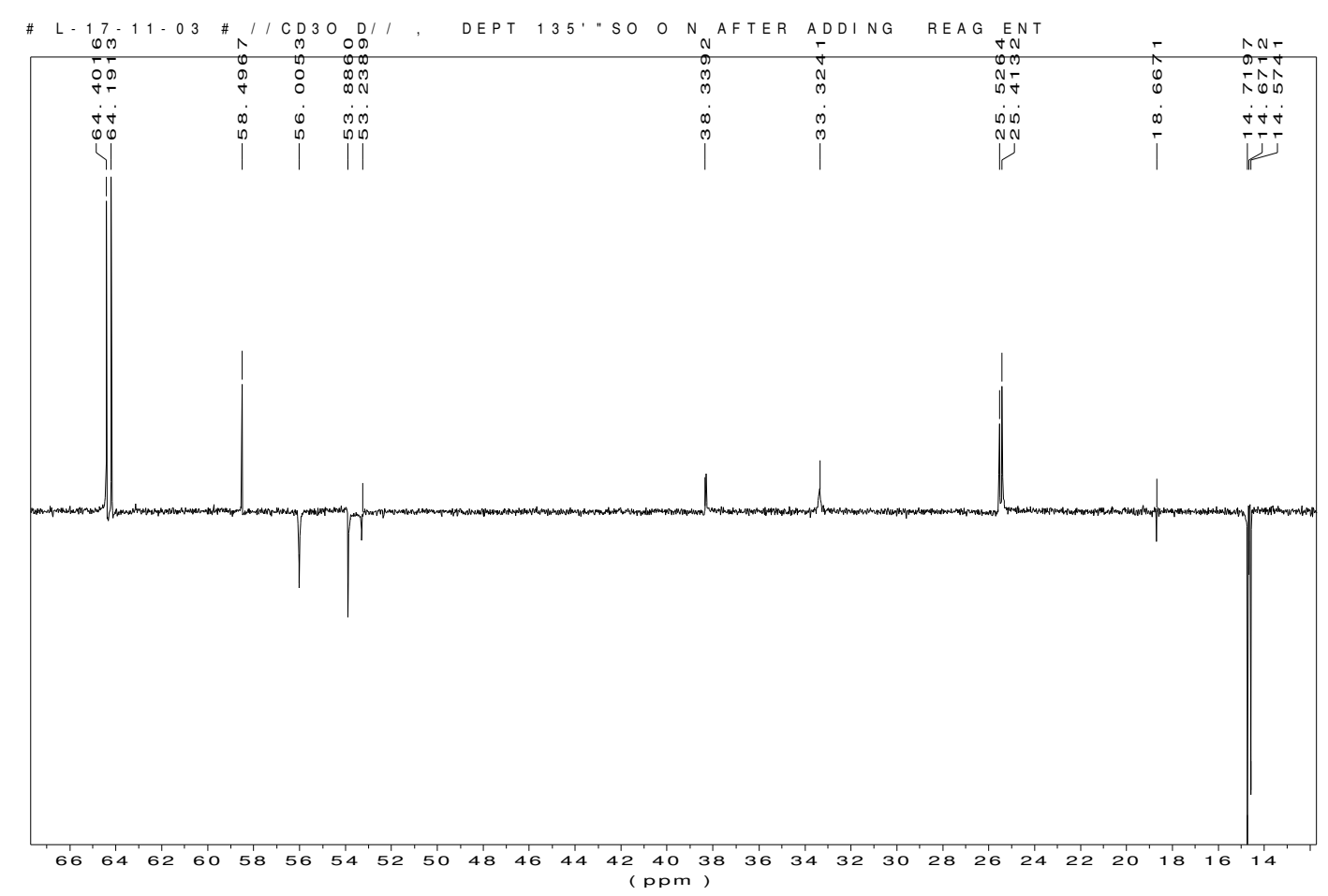

\section{${ }^{13}$ C NMR assignment:}

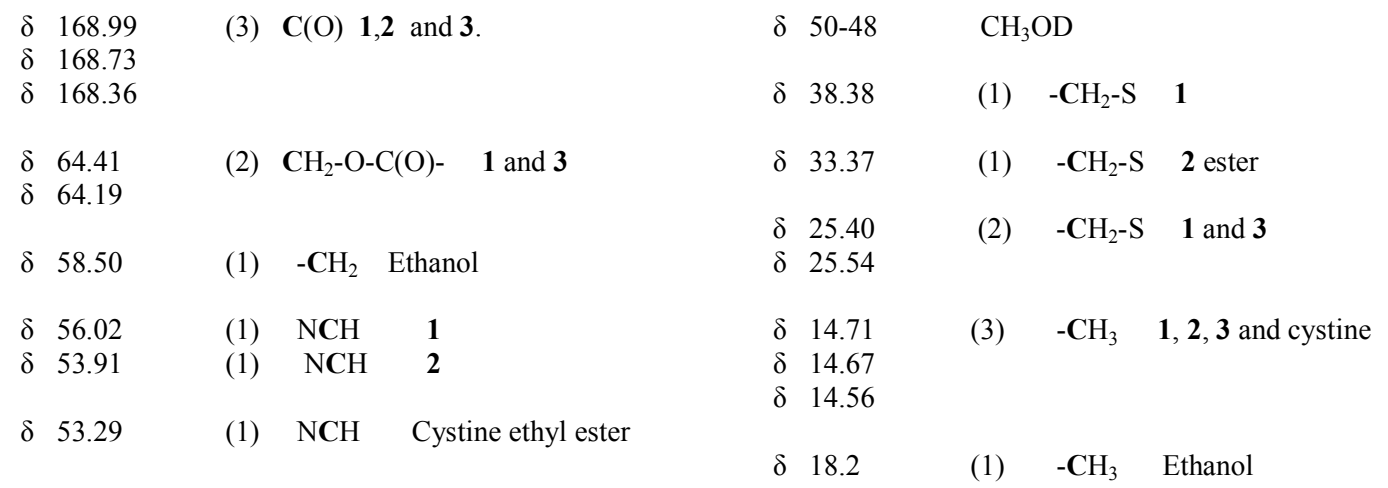




\section{Figure SI 4. HETCOR}

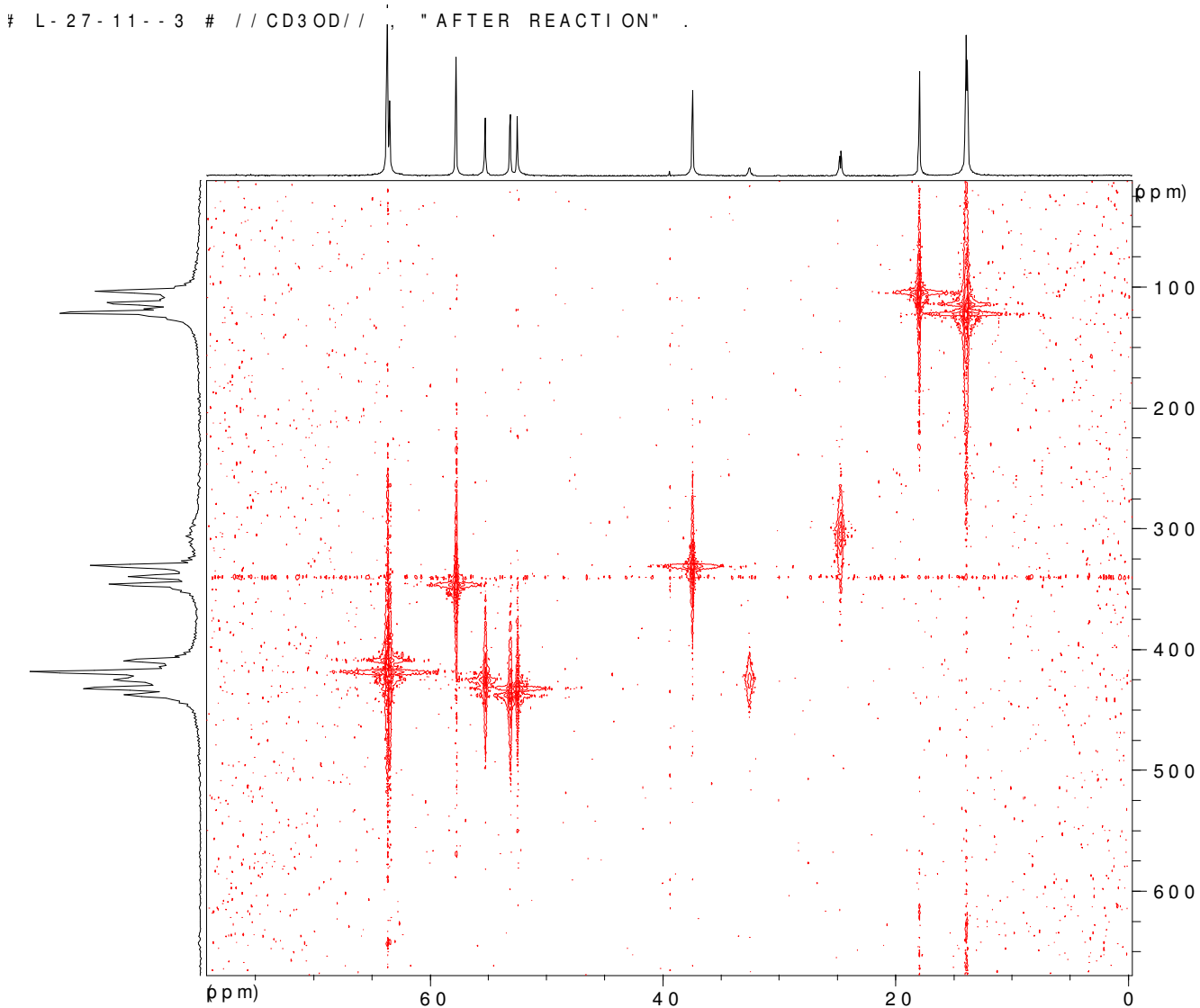

Figure SI 5. COSY

\#?? L-16-12-03 ? \# / CD3OD/ , COSY 16/12/03, "L1612PAR.FD"

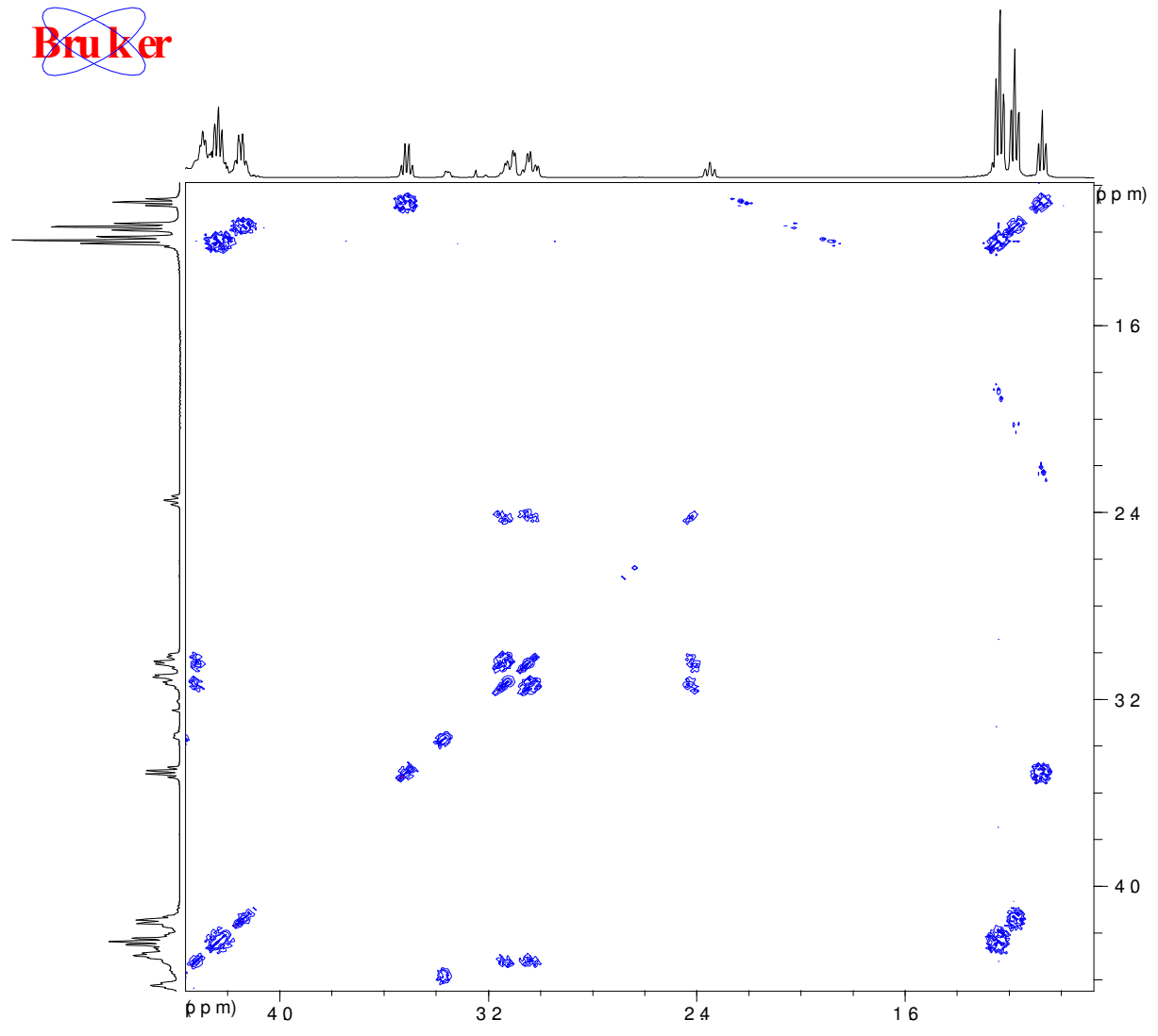




\section{Figure SI 6. Arrhenius plot}

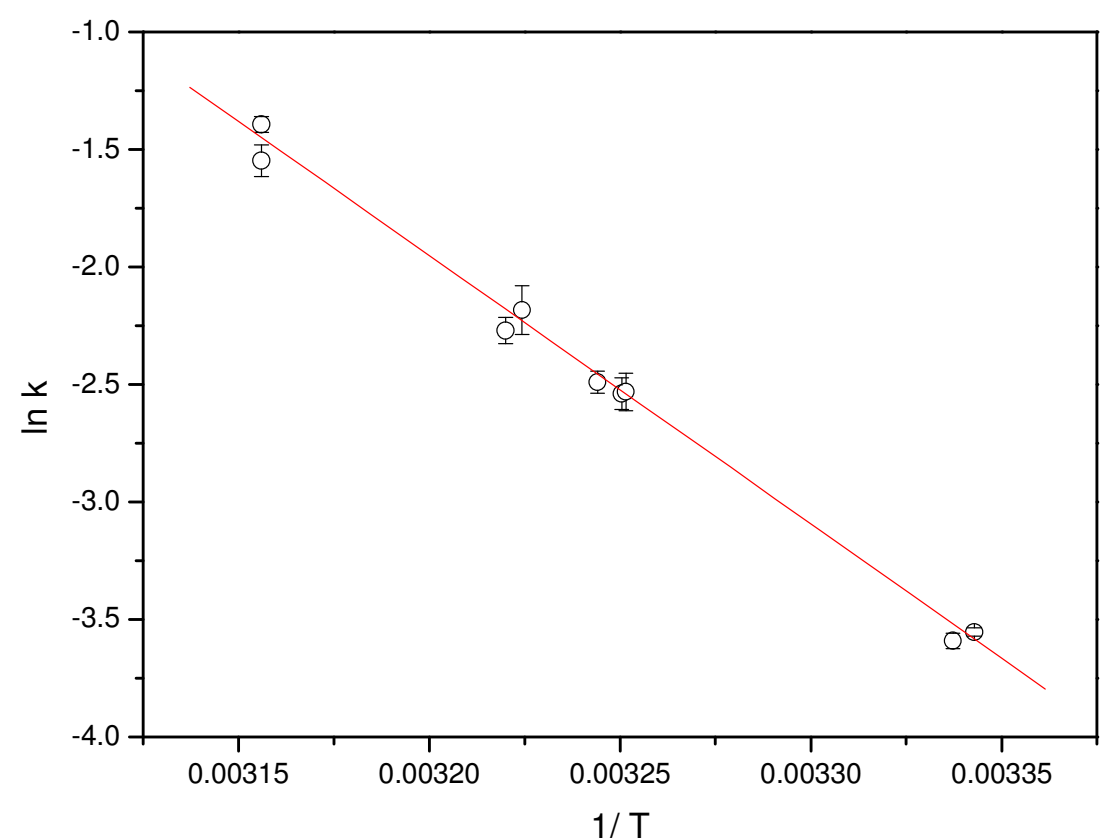

$\mathrm{Ea}=22.69 \pm 0.34 \mathrm{Kcal} / \mathrm{mol}$

$\mathrm{R}=0.99822$

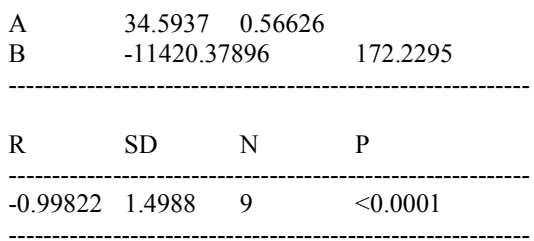

${ }^{1}$ Clancy, R.; Cederbaum, A.; Stoyanovsky, D. J. Med. Chem., 2001, 44, 2035-2038.

Complete Gaussian98 citation:

${ }^{2}$ Frisch, M. J.; Trucks, G. W.; Schlegel, H. B.; Scuseria, G. E.; Robb, M. A.; Cheeseman, J. R.; Zakrzewski, V. G.; Montgomery, J. A. Jr.; Stratmann, R.; Burant, J.; Dapprich, S.; Millam, J. M.; Daniels, A. D.; Kudin, K. N.; Strain, M. C.; Farkas, O.; Tomasi, J.; Barone, V. ; Cossi, M.; Cammi, R.; Mennucci, B.; Pomelli, C.; Adamo, C.; Clifford, S.; Ochterski,J.; Petersson, G. A.; Ayala, P. Y.; Cui, Q.; Morokuma, K.; Malick, D. K.; Rabuck, A. D.; Raghavachari, K.; Foresman, J. B.; Cioslowski, J.; Ortiz, J. V.; Baboul, A. G.; Stefanov, B. B.; Liu, G.; Liashenko, A.; Piskorz, P.; Komaromi, I.; Gomperts, R.; Martin, R. L.; Fox, D. J.; Keith, T.; Al-Laham, M. A.; Peng, C. Y.; Nanayakkara, A.; Gonzalez, C.; Challacombe, M.; Gill, P. M. W.; Johnson, B.; Chen, W.; Wong, M. W.; Andres, J. L.; Gonzalez, C.; Head-Gordon, M.; Replogle, E. S.; and Pople, J. A., Gaussian 98, Rev. A7, Gaussian, Inc., Pittsburgh PA, 1998.

${ }^{3}$ Becke, A. J. Chem. Phys. 1993, 98, 5648. Lee, C.; Yang, W.; Parr, R. Phys. Rev. B 1988, 37, 785-789.

${ }^{4}$ Peng, C.; Schlegel, H. B. Isr. J. Chem. 1993, 33, 449-54.

${ }^{5}$ East, A., L., L.; Radom, L. J.Chem Phys. 1997, 106, 6655-6674.

${ }^{6}$ Cossi, M.; Barone, V.; Cammi, R.; Tomasi, J. Chem. Phys. Lett. 1996, 255, 327-335. 